\title{
Combination ledipasvir-sofosbuvir for the treatment of chronic hepatitis $C$ virus infection: a review and clinical perspective
}

This article was published in the following Dove Press journal:

Therapeutics and Clinical Risk Management

3 June 2016

Number of times this article has been viewed

\author{
Marcel Nkuize' \\ Thomas Sersté ${ }^{1,2}$ \\ Michel Buset ${ }^{\prime}$ \\ Jean-Pierre Mulkay \\ 'Department of Gastroenterology \\ and Hepatology, Saint-Pierre \\ University Hospital, ${ }^{2}$ Department \\ of Gastroenterology, Pancreatology \\ and Hepatology, Hôpital Academique \\ Erasme, Université Libre de Bruxelles, \\ Brussels, Belgium
}

\begin{abstract}
Chronic hepatitis $\mathrm{C}$ treatment has continued to evolve, and interferon-free, oral treatment with direct-acting antiviral agents is the current standard of care. Recently, a new treatment, which is a combination of two direct-acting antiviral agents, ledipasvir $90 \mathrm{mg}$ (anti-NS5A) and sofosbuvir $400 \mathrm{mg}$ (anti-NS5B), has been approved in the US and the European Union for the treatment of chronic hepatitis $\mathrm{C}$ viral infection. In Phase III trials among chronic hepatitis $\mathrm{C}$ virus genotype 1 monoinfected (treatment-naïve, treatment-experienced, and with advanced liver disease or posttransplant) patients and HIV-hepatitis C virus coinfected patients, the ledipasvir-sofosbuvir fixed-dose combination is associated with a higher rate of sustained virologic response at 12 weeks after therapy has ceased. According to preliminary data, the ledipasvir-sofosbuvir combination also may be effective against hepatitis $C$ genotype 4 virus infection. The ledipasvir-sofosbuvir combination taken orally is generally well-tolerated. Moreover, the combination treatment may suppress the effect of predictive factors of chronic hepatitis $\mathrm{C}$ that have historically been known to be associated with treatment failure. Thus, the fixed-dose single-tablet combination of ledipasvir-sofosbuvir offers a new era for the effective treatment of a variety of patients suffering from chronic hepatitis $\mathrm{C}$ virus infection.
\end{abstract}

Keywords: ledipasvir, liver disease, ethnicity, DAA, HIV

\section{Introduction}

Chronic hepatitis $\mathrm{C}(\mathrm{CHC})$ affects more than 170 million people worldwide. ${ }^{1}$ Its complications, such as cirrhosis and hepatocellular carcinoma, can be resolved by liver transplantation, but liver grafts can often become reinfected with hepatitis $\mathrm{C}$ virus (HCV) ${ }^{2,3} \mathrm{HCV}$-related end-stage liver disease that necessitates liver transplantation and graft recidivism due to $\mathrm{HCV}$ infection (causing graft loss in 30\%) can be avoided by effective treatment of HCV-infected patients. Until 2010, the worldwide standard of care for these patients was the combination of subcutaneous pegylated-interferon (IFN) and oral ribavirin (RBV), which provided an overall cure rate of only slightly more than half of all HCV-infected patients. ${ }^{3,4}$ In addition, this standard treatment was accompanied by several adverse effects (eg, flu-like syndrome, depression, and cytopenia for pegylated-IFN and hemolytic anemia, rash, pruritus, and insomnia for RBV) leading to a poor quality of life for patients. ${ }^{3}$ In 2011, addition of protease inhibitors to pegylated-IFN plus RBV therapy was associated with an increase in the cure rate to $\sim 65 \%$ of $\mathrm{HCV}$ genotype 1 (HCV-1)-infected recipients, but adverse events and treatment discontinuation were still very common. ${ }^{4-6}$ While the standard of care for $\mathrm{CHC}$ is still IFN-based in lower income countries, since 2012-2013 CHC treatment in higher income countries has moved into a new era of direct-acting antiviral agents (DAAs),
Correspondence: Marcel Nkuize

Department of Gastroenterology and Hepatology, Saint-Pierre University Hospital, 322 Rue Haute, 1000 Brussels, Belgium

Tel +32 25353111

Fax +32 25353686

Email marcelnkuize@hotmail.com 
which are oral, IFN-free molecules that target $\mathrm{HCV}$ proteins. ${ }^{4,5,7}$ Several DAAs (eg, simeprevir, sofosbuvir [SOF]) are already used in clinical practice and others are either being evaluated in clinical trials or are under development. ${ }^{4,5,8-10}$ Recently, in October 2014, the US Food and Drug Administration approved the combination of ledipasvir and SOF (LDV/SOF, brand name Harvoni, Gilead Sciences in the US [Foster City, CA, USA] and EU) for the treatment of $\mathrm{CHC} .{ }^{10,11}$ In this review, we will summarize current knowledge of the pharmacodynamics and pharmacokinetics of LDV/SOF, and review the results of Phase III trials of the LDV/SOF combination. We will also provide our clinical perspective on this therapy.

\section{LDVISOF pharmacodynamic properties}

The LDV/SOF combination is administered as a single tablet containing a fixed-dose of $90 \mathrm{mg}$ LDV and $400 \mathrm{mg} \mathrm{SOF} .{ }^{11}$

\section{Mechanism of action}

LDV is active against $\mathrm{HCV}$ NS5A, a protein involved in $\mathrm{HCV}$ replication, assembly, and secretion. SOF is a pyrimidine nucleotide analogue that inhibits HCV NS5B RNA-dependent RNA polymerase, the key enzyme mediating HCV RNA replication. HCV NS5A and NS5B RNA-dependent RNA polymerases are crucial for $\mathrm{HCV}$ replication. ${ }^{11-13}$

\section{Antiviral activity}

LDV has different degrees of antiviral activity across all HCV genotypes. ${ }^{11,12}$ The 50\% effective inhibitory concentration $\left(\mathrm{EC}_{50}\right)$ has potent activity for $\mathrm{HCV}-1$ (HCV-1a, $\mathrm{HCV}-1 \mathrm{~b})$; moderate activity for HCV genotypes 4 (HCV-4), 5 (HCV-5), and 6a (HCV-6a); and lower activity for HCV genotypes $2 \mathrm{a}(\mathrm{HCV}-2 \mathrm{a}), 3 \mathrm{a}(\mathrm{HCV}-3 \mathrm{a})$, and 6e (HCV-6e). ${ }^{1-14}$ SOF has higher antiviral activity than LDV against all HCV genotypes. ${ }^{15}$

\section{Resistance}

Resistance to LDV and SOF has been identified and was attributed to an amino acid substitution at a specific-site in HCV NS5A and NS5B, respectively. ${ }^{11-14}$

In cell culture, reduced susceptibility to LDV has been observed and was attributed to primary NS5A substitutions Y93H and Q30E, for HCV-1a, and Y93H for HCV-1b. These resistance-associated variants (RAVs) led to a 100- to 1,000-fold change in LDV susceptibility. ${ }^{12}$ In Phase III clinical trials, the most frequent substitutions found at treatment failure were Q30R, Y93H/N, and L31M for HCV-1a, and Y93H for HCV-1b. ${ }^{9,16-20}$
In cell culture, reduced susceptibility to SOF (2- to 18-fold) was related to S282T substitution only; SOF is a drug with a high genetic barrier. ${ }^{11}$ This S282T mutation was not found in Phase III trials using LDV/SOF for HCV treatment. ${ }^{11}$ There is very limited impact of RAVs identified at the start of LDV/SOF course given the very high cure rate of HCV-1infected patients treated with LDV/SOF. ${ }^{9,11,16-18,20}$

There is no cross-resistance between LDV and SOF, meaning that LDV has excellent antiviral activity against SOF-induced mutants, while SOF is active against LDVinduced mutations. ${ }^{11} \mathrm{LDV}$ and SOF have antiviral activity against RAVs associated with NS5B nonnucleoside and NS3 protease inhibitors. ${ }^{11}$

\section{LDV/SOF pharmacokinetic properties}

One LDV/SOF fixed-dose combination tablet is taken orally once daily with or without food. After oral administration of one LDV/SOF tablet, the median peak plasma concentrations of LDV, SOF, and GS-331007 (the predominant circulating inactive metabolite of SOF) are reached after $4, \sim 1$, and 4 hours, respectively. ${ }^{11,12}$

LDV and SOF have $99.8 \%$ and $63 \%$ plasma protein binding, whereas GS-331007 binding to plasma proteins is minimal. The median terminal half-lives are 47, 0.5, and 27 hours for LDV, SOF, and GS-331007, respectively. ${ }^{11}$

LDV is metabolized through slow oxidative metabolism and SOF is metabolized through the liver into the active metabolite GS-461203 before dephosphorylation into the inactive metabolite GS-331007 which represents $85 \%$ of total systemic exposure after administration of LDV/SOF. Routes of clearance are biliary for LDV and renal for GS-331007. ${ }^{11,12}$

There is no need to modify LDV/SOF dosage for patients with mild or moderate renal insufficiency. GS-331007 concentrations increase in severe renal insufficiency (glomerular filtration rate $<30 \mathrm{~mL} / \mathrm{min} / 1.73 \mathrm{~m}^{2}$ ) or end-stage renal disease and $\mathrm{LDV} / \mathrm{SOF}$ should be avoided in these patients. $^{11,12,21}$

There is no need to modify LDV/SOF dosage in cases of moderate to severe hepatic insufficiency, and demographic parameters (age, body weight, sex) and ethnicity do not modify the pharmacokinetics of LDV/SOF or GS-331007. ${ }^{11,12,14,21-23}$

\section{Drug interactions}

LDV and SOF are substrates of P-glycoprotein transporters. ${ }^{11-14}$ Inducers of P-glycoprotein (eg, rifampicin, St John's wort, 
carbamazepine, phenytoin, and tipranavir [ritonavir boosted]) may decrease intestinal absorption of LDV/SOF leading to decreased efficacy. Other inducers potentially associated with significant interaction with LDV/SOF include digoxin, dabigatran etexilate, rosuvastin, simeprevir, and tenofovir disoproxil fumarate when associated with pharmacologic enhancers such as ritonavir. ${ }^{10-12,22,23}$ There are no clinically significant interactions between LDV/SOF and abacavir, atazanavir/ritonavir, cyclosporine, darunavir/ritonavir, emtricitabine, efavirenz, lamivudine, methadone, oral contraceptives, pravastatin, rategravir, rilpivirine, tacrolimus, tenofovir disoproxil fumarate, or verapamil. ${ }^{11-14,24-26}$

LDV solubility decreases as $\mathrm{pH}$ increases, so that drugs that increase gastric acidity (eg, antacids, proton pump inhibitors) may decrease plasma concentrations of LDV. Proton pump inhibitors should not be taken before LDV/SOF. Twenty milligrams of proton pump inhibitor equivalent to omeprazole $20 \mathrm{mg}$ can be safely administrated at the same time or $>2$ hours after LDV/SOF. ${ }^{11-14,25,26}$

\section{Summary of Phase III clinical trials with the LDV/SOF combination in HCV-infected patients LDV/SOF in chronic HCV monoinfected patients \\ LDV/SOF in chronic HCV-I infection}

Three major trials (ION-1, -2, and -3) (Table 1) have been conducted in chronic HVC-1 infection therapy.,16,17 They were all Phase III, open-label, randomized trials. The aim of the trials was to evaluate the efficacy and safety of a fixed-dose of the LDV/SOF combination among different groups of recipients.

In ION-1, 865 treatment-naïve (TN) patients (including $16 \%$ with cirrhosis, $12 \%$ of African-American origin, and $67 \%$ of $\mathrm{HCV}-1 \mathrm{a}$ ) were randomized to receive $\mathrm{LDV} /$ SOF alone for 12 weeks (group A), LDV/SOF plus RBV for 12 weeks (group B), LDV/SOF alone for 24 weeks (group C), or LDV/SOF plus RBV for 24 weeks (group D). ${ }^{9}$ The primary endpoint was sustained virologic response 12 weeks off therapy (SVR12) (which is defined by HCV RNA $<$ lower limit of quantification, analyzed by $\mathrm{COBAS}^{\circledR}$ TaqMan $^{\circledR}$ HCV Test v2.0 HPS, with lower limit of quantification of $25 \mathrm{IU} / \mathrm{mL})$. SVR12 rates were 99\% (95\% confidence interval [CI] 96-100), 97\% (95\% CI 94-99), 98\% (95\% CI 95-99), and 99\% (95\% CI 97-100) in groups A, B, C, and D, respectively. There were no improvements in SVR12 when RBV was given concurrently with LDV/SOF. Three patients experienced virologic failure, two relapsed and had NS5A-RAVs at baseline, whereas one patient with virologic breakthrough did not; one HCV-1a patient had L31M mutation and one HCV-1b had L31M and Y93H. There were no NS5B-RAVs found either at baseline or at any time during treatment. The most common adverse events were headache, fatigue, insomnia, and nausea. None of the patients receiving LDV/SOF for 12 weeks needed to have the drug discontinued (groups A and B). In conclusion, the study found that the LDV/SOF combination was highly effective among HCV-1infected $\mathrm{TN}$ recipients.

ION-2 included 440 patients who had not been cured by previous therapy (treatment-experienced [TE]); 20\% were cirrhotic, and $79 \%$ were HCV-1a. ${ }^{16}$ They were randomized

Table I LDV/SOF Phase III trials for the treatment of patients with chronic hepatitis C genotype I infection

\begin{tabular}{|c|c|c|c|c|c|c|c|}
\hline Trial & Patient characteristics & $\begin{array}{l}\text { Treatment } \\
\text { regimen }\end{array}$ & $\begin{array}{l}\text { Number } \\
\text { of pts }\end{array}$ & $\begin{array}{l}\text { Duration } \\
\text { (weeks) }\end{array}$ & SVR I 2 (\%) & Relapse (\%) & LTF/WC \\
\hline \multirow[t]{4}{*}{ ION-I9 } & Treatment-naïve & LDV/SOF & $214^{*}$ & 12 & $210 / 213(99)$ & $1 / 212(<\mathrm{I})$ & $2 / 213 / 0$ \\
\hline & & LDV/SOF + RBV & 217 & 12 & $211(97)$ & 0 & $4 / 2$ \\
\hline & & LDV/SOF & 217 & 24 & $212(98)^{\#}$ & $\mathrm{I}(<\mathrm{I})$ & $2 / 1$ \\
\hline & & $\mathrm{LDV} / \mathrm{SOF}+\mathrm{RBV}$ & $217^{*}$ & 24 & $215(99)$ & 0 & $2 / 0$ \\
\hline \multirow[t]{4}{*}{$1 O N-2^{16}$} & Treatment-experienced & LDV/SOF & 109 & 12 & $102(94)$ & $7(6)$ & $0 / 0$ \\
\hline & & LDV/SOF + RBV & 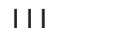 & 12 & $107(96)$ & $4(4)$ & $0 / 0$ \\
\hline & & LDV/SOF & 109 & 24 & $108(99)$ & 0 & $0 / 0$ \\
\hline & & LDV/SOF + RBV & III & 24 & $110(99)^{\#}$ & 0 & $0 / 0$ \\
\hline \multirow[t]{3}{*}{$10 N-3^{17}$} & Treatment-naïve & LDV/SOF & 215 & 8 & $202(94)$ & II (5) & $1 / 1$ \\
\hline & & $\mathrm{LDV} / \mathrm{SOF}+\mathrm{RBV}$ & 216 & 8 & $201(93)$ & $9(4)$ & $5 / 1$ \\
\hline & & LDV/SOF & 216 & 12 & $208(95)$ & $3(1)$ & $7 / 0$ \\
\hline \multirow[t]{2}{*}{ Japan ${ }^{18}$} & Treatment-naïve or -experienced & LDV/SOF & |7| & 12 & 100 & 0 & 0 \\
\hline & & $\mathrm{LDV} / \mathrm{SOF}+\mathrm{RBV}$ & 170 & 12 & 98 & $\mathrm{I}(<\mathrm{I})$ & $* *$ \\
\hline
\end{tabular}

Notes: *One patient excluded because of HCV GT4 infection, ${ }^{*}$ one virologic failure during treatment, **one patient discontinued from the study on day 6 because of adverse events and one patient discontinued from the study on day 62 because of adverse events and died the next day.

Abbreviations: HCV, hepatitis C virus; LDV/SOF, ledipasvir and sofosbuvir; pts, patients; RBV, ribavirin; SVRI2, sustained virologic response I2 weeks off therapy; LTF/ WC lost to follow-up/withdrew consent. 
to receive $\mathrm{LDV} / \mathrm{SOF}$ for 12 weeks (group I), LDV/SOF plus RBV for 12 weeks (group II), LDV/SOF for 24 weeks (group III), or LDV/SOF plus RBV for 24 weeks (group IV). The SVR12 rate was 94\% (95\% CI 87-97) in group I; 96\% (95\% CI 91-97) in group II; 99\% (95\% CI 95-100) in group III; and 99\% (95\% CI 95-100) in group IV. In the 12-week treatment group, the subgroup of cirrhotic patients had a modestly lower SVR12 rate compared with noncirrhotic patients, whereas there was no difference in SVR12 rates among cirrhotic and noncirrhotic patients who had received 24 weeks of treatment. The study was not designed to compare SVR12 rates between types of treatment or duration of treatment. Relapses were observed only among recipients who had received 12 weeks of treatment, in contrast to the recipients who had received 24 weeks of treatment without a relapse. Among seven patients in the group treated with LDV/SOF for 12 weeks who had a relapse, four had NS5ARAVs at baseline. Of four patients in the group treated with LDV/SOF plus RBV for 12 weeks who had a relapse, two had NS5A-RAVs at baseline. Therefore, RAVs were found in all of the eleven patients at the time of the relapse. No NS5B-RAVS were found either at baseline or at any time during treatment. In conclusion, the study found that the LDV/SOF combination was highly effective among HCV-1infected recipients for whom standard treatment had previously failed.

ION-3 included $647 \mathrm{TN}$ patients without cirrhosis who were randomized to receive LDV/SOF for 8 weeks (group F), LDV/SOF plus RBV for 8 weeks (group G), or LDV/SOF for 12 weeks (group H). ${ }^{17}$ The SVR12 rate was 94\% (95\% CI 90-97) in group F, 93\% (95\% CI 89-96) in group G, and $95 \%$ (95\% CI 92-98) in group H. In post hoc analysis, in the subgroup of patients who had HCV RNA $<6$ million IU/mL, SVR12 rates were $97 \%$ for patients treated with $\mathrm{LDV} / \mathrm{SOF}$ alone for 12 weeks, $96 \%$ for treatment with $\mathrm{LDV} / \mathrm{SOF}$ plus RBV for 8 weeks, and $96 \%$ for treatment with LDV/ SOF plus RBV for 12 weeks. ${ }^{15,27}$ Of 647 patients treated, 23 (3.5\%) patients had a relapse. NS5A-RAVs were present in 15 patients at the time of relapse (and at baseline in 9/15 [60.0\%]). No NS5B-RAVs were found either at baseline or at any time during treatment. In conclusion, the study found that HCV-1-infected patients without cirrhosis who received 8 weeks of LDV/SOF combination treatment achieved high SVR12 rates.

A Japanese open-label, multicenter, Phase III trial included $341 \mathrm{TN}$ and TE patients (Table 1) who were randomized to receive LDV/SOF for 12 weeks (group I) or LDV/SOF plus RBV for 12 weeks (group II). ${ }^{18} \mathrm{~A}$ total of
171 patients were included in group I and 170 were included in group II. SVR12 was attained by 171 (100\%) patients (83/83 TN and 88/88 TE patients) who received LDV/SOF and $167 / 170(98 \%)$ patients (80/83 TN and 87/87 TE) who received LDV/SOF plus RBV. Of the 170 patients treated with LDV/SOF plus RBV, two (1.2\%) who were cirrhotic had a treatment discontinuation due to adverse events. The most frequent adverse events were nasopharyngitis and headache in both groups and anemia in the LDV/SOF plus RBV group. Of the 76 patients who had NS5A-RAVs at baseline, 75 (99\%) achieved SVR12. In conclusion, the study found that the fixed-dose LDV/SOF combination offered an important option for treatment of Japanese patients suffering from $\mathrm{HCV}-1$ infection.

The ION and Japanese trials illustrated that LDV/SOF was highly efficient in the treatment of HCV-1 infection in $\mathrm{TN}$ patients as well as in TE patients. In the ION trials, 1,456/1,518 (95.9\%) of patients achieved SVR12.9,16,17 Moreover, compensated cirrhotic and noncirrhotic patients similarly achieved high SVR12 rates (see "Impact of negative predictors of SVR derived from IFN-based therapy" section). Relapse was very uncommon (2\%) and was $5 \%$, $2 \%$, and $0.2 \%$ in patients treated with $\mathrm{LDV} / \mathrm{SOF}$ for 8,12 , and 24 weeks, respectively. The results from the Japanese trial found a similar pattern of SVR rates. The treatment was well-tolerated and the most common adverse effects, nausea, headache, insomnia, and anemia, were more frequent when $\mathrm{RBV}$ was added to $\mathrm{LDV} / \mathrm{SOF}$.

\section{LDV/SOF in chronic HCV-2, -5, or -6 infections}

No major trials have been conducted in chronic HCV-2 and -6 infection. For HCV-5 infection, only an abstract is available, which was presented during the European Association for the Study of Liver (EASL) congress in 2015. ${ }^{28}$ This multicenter study was carried out in France, and included TN ( $n=21)$ and TE $(n=20)$ chronic HCV-5-infected patients. Patients were treated with LDV/SOF for 12 weeks. The SVR12 rate was 20/21 (95\%) and 19/20 (95\%) in TN and TE patients, respectively. Nine patients had cirrhosis (three in $\mathrm{TN}$ and six in TE), and the SVR12 rate was $8 / 9(88.8 \%)$ in these patients compared to $31 / 32(96.8 \%)$ in those without cirrhosis. No patient had treatment discontinuation related to adverse events. In conclusion, LDV/SOF was well-tolerated and highly effective without the need for RBV.

\section{LDV/SOF in chronic HCV-3 infection}

In the open-label, Phase II ELECTRON-2 study, one arm included patients with $\mathrm{HCV}-3$ infection. ${ }^{29,30}$ In this group, 
$\mathrm{TN}$ patients were randomized to receive $\mathrm{LDV} / \mathrm{SOF}$ alone for 12 weeks ( $n=25)$ (group A) or LDV/SOF plus RBV for 12 weeks ( $\mathrm{n}=26)$ (group B), and TE patients were treated with LDV/SOF plus RBV for 12 weeks $(n=50)$ (group C). The SVR12 rate was $64 \%$ and $100 \%$ in groups A and B, respectively, and $82 \%$ in group $\mathrm{C}$. This early result suggests that LDV/SOF is likely to be less effective in HCV-3 infection; this finding could be anticipated from the $\mathrm{EC}_{50}$ of the combination against HCV-3 which was very high compared with HCV-1 infection (see ION trials). ${ }^{9,16,17}$ It is not clear whether larger trials might increase this SVR12 rate. Perhaps association with drugs targeting other HCV particles may be an option for further study.

\section{LDV/SOF in chronic HCV-4 infection}

There has been no major trial of treatment of HCV-4-infected patients with the LDV/SOF combination. A small number of chronic HCV-4-infected patients (42/659 [6\%]) have been included in other studies (see "LDV/SOF in chronic HCV-1 or -4-infected patients with advanced liver disease and posttransplant" section).

An open-label, single-center Phase IIa trial of LDV/SOF treatment in HCV-4-infected patients (the NIAID Synergy study) recruited 21 patients (14 male, nine Black-Africans, six Egyptians/five Americans, four Ethiopians, three Cameroonians) of whom 13 had HCV RNA > 800,000 IU/mL, eight were IFN TE, and seven had cirrhosis (evaluated by Fibrosure test plus aspartate aminotransferase-to-platelet). ${ }^{31,32}$ Patients who were coinfected with HIV or hepatitis B virus, or had decompensated cirrhosis or previous DAA treatment experience were excluded. The aim of the study was to assess the efficacy (evaluated by the SVR12 measured with COBAS TaqMan HCV test version 1.0, with a lower limit of quantification of $43 \mathrm{IU} / \mathrm{mL}$ ), safety, and tolerability of LDV/SOF in patients treated for 12 weeks. The SVR12 rate was 19/20 (the single patient without SVR12 was noncompliant with the treatment). ${ }^{31,32}$ No treatment discontinuations occurred because of adverse events and there were no grade 2 or 3 adverse events during the 12 weeks of LDV/SOF treatment. The most common adverse events were diarrhea (two), nausea (two), upper respiratory infection (two), and fatigue (three).

Another multicenter study tested LDV/SOF treatment for 12 weeks in TN $(n=22)$ and TE $(n=22)$ HCV-4-infected patients in France. ${ }^{28}$ SVR12 rates were 21/22 (95.4\%) and 20/22 (90.9\%) in TN and TE patients, respectively. Ten patients had cirrhosis (one TN and nine TE), and the SVR12 rate was $10 / 10(100.0 \%)$ in cirrhotic patients versus $31 / 34$
$(91.1 \%)$ in noncirrhotic patients. There was no treatment discontinuation due to adverse events. LDV/SOF was welltolerated and associated with high SVR12 rates without the need for RBV.

\section{LDV/SOF in chronic HCV-I or -4-infected patients with advanced liver disease and posttransplant}

Studies on the efficacy and safety of DAA in patients with advanced cirrhosis and in liver-transplant recipients with recurrent $\mathrm{HCV}$ infection are scarce. ${ }^{33-35}$

The efficacy and safety of LDV/SOF for the treatment of chronic HCV infection and advanced liver disease have been assessed in an open-label Phase II study (Table 2) in patients with decompensated cirrhosis or posttransplant recurrence (Clinicaltrials.gov identifier NCT01938430, the SOLAR-1 study). ${ }^{34}$ The study included two cohorts: cohort A consisted of patients suffering from cirrhosis and advanced liver disease. Two separate groups were identified within this cohort: patients with Child-Pugh B cirrhosis (Child score from 7 to 9) and patients with Child-Pugh C cirrhosis (Child score from 10 to 12 ). A second cohort, cohort B, included patients with liver transplantation and posttransplant $\mathrm{HCV}$ recurrence. Five separate groups were identified in this posttransplant cohort: patients without cirrhosis and various levels of fibrosis (from F0 to F3); patients with fibrosing cholestatic hepatitis; and patients with Child-Pugh A, B, and $\mathrm{C}$ cirrhosis. All patients in both cohorts were randomly allocated in a $1: 1$ ratio to receive 12 or 24 weeks of $\mathrm{LDV} / \mathrm{SOF}$ plus RBV for 12 or 24 weeks.

In cohort A (nontransplanted patients), 108 subjects were randomized (two patients were HCV-4-infected) ${ }^{34}$ The Child-Pugh score varied from 7 to 12 . The vast majority of patients in this cohort had ascites and encephalopathy. A dose-escalation regimen was proposed for RBV administration. The SVR rates were similar with 12 or 24 weeks of LDV/SOF plus RBV. Indeed, in the Child-Pugh B group, SVR12 was achieved by $87 \%$ (12 weeks arm) and $89 \%$ (24 weeks arm) of patients. In the Child-Pugh $\mathrm{C}$ group, SVR12 was achieved in 86\% (12 weeks arm) and 90\% (24 weeks arm) of patients. The SVR12 rate was associated with an improvement in bilirubin and albumin concentrations and in a model for end-stage liver disease scores in both groups. Serious adverse events occurred in 30 patients. This SVR rate was high given that the population was suffering from advanced liver disease and, in most cases, anemia, hepatic encephalopathy, and peritoneal hemorrhage. Six patients died during the study in this group, mostly related to septic shock. No direct relationship between those deaths 
Table 2 SVRI 2 rates in advanced liver disease and postliver transplant treated with LDV/SOF + RBV

\begin{tabular}{|c|c|c|c|c|}
\hline Trial & Treatment duration, number & SVRI 2 rate & $95 \% \mathrm{Cl}$ & BKT/REL \\
\hline \multicolumn{5}{|l|}{ SOLAR-I ${ }^{34}$} \\
\hline \multicolumn{5}{|c|}{ Pretransplant Cohort A } \\
\hline \multirow[t]{2}{*}{ CTP.B } & 12 weeks $(n=30)$ & $26 / 30(87)$ & $72-95$ & $0 / 3$ \\
\hline & 24 weeks ( $n=29)$ & $24 / 27(89)$ & $74-97$ & $0 / 1$ \\
\hline \multirow[t]{2}{*}{ CTP.C } & 12 weeks $(n=23)$ & $19 / 22(86)$ & $68-96$ & $0 / 1$ \\
\hline & 24 weeks $(n=26)$ & $20 / 23(87)$ & $70-96$ & $0 / 2$ \\
\hline \multicolumn{5}{|c|}{ Posttransplant Cohort B } \\
\hline No cirrhosis & 12 weeks $(n=55)$ & $53 / 55(96)$ & 89-99 & $0 / 2$ \\
\hline Group III & 24 weeks $(n=56)$ & $56 / 56(98)$ & $92-100$ & $0 / 0$ \\
\hline CTP.A & 12 weeks $(n=26)$ & $25 / 26(96)$ & $83-100$ & $0 / 0$ \\
\hline Group IV & 24 weeks $(n=25)$ & $24 / 25(96)$ & $82-100$ & $0 / 0$ \\
\hline CTP.B & 12 weeks $(n=26)$ & $22 / 26(85)$ & $68-95$ & $0 / 1$ \\
\hline Group V & 24 weeks $(n=26)$ & $23 / 26(88)$ & $73-97$ & $0 / 0$ \\
\hline CTP.C & 12 weeks $(n=5)$ & $3 / 5(60)$ & 19-92 & $0 / 2$ \\
\hline Group VI & 24 weeks $(n=4)$ & $3 / 4(75)$ & $25-99$ & $0 / 1$ \\
\hline Fib. chol. & I 2 weeks $(n=4)$ & $4 / 4(100)$ & $47-100$ & $0 / 0$ \\
\hline Group VII & 24 weeks $(n=2)$ & $2 / 2(100)$ & $22-100$ & $0 / 0$ \\
\hline \multicolumn{5}{|l|}{ SOLAR-2 ${ }^{36}$} \\
\hline Posttransplant & 12 weeks $(n=86)$ & $82 / 86(95)$ & NA & $\mathrm{NA} / \mathrm{I}$ \\
\hline (F0-F3 + CTP.A) & 24 weeks $(n=82)^{*}$ & $64 / 65(98)$ & NA & $\mathrm{NA} / 0$ \\
\hline Pre-/posttransplant & 12 weeks $(n=78)$ & $61 / 72(85)$ & NA & $\mathrm{NA} / 7$ \\
\hline (CTP.B/C) & 24 weeks $(n=82)^{*}$ & $60 / 68(88)$ & NA & $\mathrm{A} / 2$ \\
\hline
\end{tabular}

Notes: $* 27$ patients in the 24 week arm did not reach SVRI2; ten patients did not meet inclusion criteria (seven patients who were transplanted + three patients without inclusion criteria) and were excluded.

Abbreviations: BKT/REL, breakthrough/relapse; $95 \% \mathrm{Cl}, 95 \%$ confidence interval; CTP A, B, C, Child-Trucotte-Pugh of class A, B and C; F, fibrosis score according to the METAVIR classification; Fib. chol., fibrosing cholangitis; LDV/SOF, ledipasvir and sofosbuvir; NA, not applicable; RBV, ribavirin; SVRI2, sustained virologic response I2 weeks off therapy.

and the treatment was identified. No on-treatment virological failures were observed.

In cohort B (transplanted patients), 223 subjects were randomized to 12 or 24 weeks of treatment (three patients were HVC-4-infected). ${ }^{34}$ All had been transplanted for more than 3 months prior to the study. No drug-drug interactions were expected with tacrolimus or cyclosporine. In patients with posttransplant cirrhosis and Child-Pugh B and C scores, a dose escalation for RBV was proposed as in cohort A. In the vast majority of patients (from $78 \%$ to $90 \%$, according to group), previous HCV treatment had been offered. SVR12 rates were 96\% (12 weeks arm) and 98\% (24 weeks arm) in patients with F0-F3 fibrosis. All patients with fibrosing cholestatic hepatitis achieved SVR12. In patients with ChildPugh A cirrhosis, SVR12 rate was 96\% (both 12 weeks and 24 weeks arms). In patients with advanced cirrhosis, the SVR12 rates were $85 \%$ (12 weeks arm) and 83\% (24 weeks arm) in Child-Pugh B patients. SVR rates were $60 \%$ (12 weeks arm) and 67\% (24 weeks arm) in Child-Pugh C patients. Serious adverse events occurred in 44 patients and mostly consisted of dyspnea and peritoneal hemorrhage. Four patients died during the study in this group, mostly related to complications of cirrhosis. No direct relationship between these deaths and the treatment was identified. No viable RAVs were detected in any of the patients who did not achieve SVR.

In conclusion, in patients with HCV-1- and -4-related cirrhosis, LDV/SOF plus RBV for 12 weeks resulted in high SVR12 rates even in the context of advanced liver disease. In patients with posttransplant HCV recurrence, treatment with LDV/SOF plus RBV was associated with high SVR12 rates, irrespective of the severity of the liver disease. Extending treatment duration to 24 weeks did not increase the response rates. LDV/SOF plus RBV for 12 or 24 weeks was generally well-tolerated with low rates of drug-related adverse events or treatment discontinuations. The LDV/SOF plus RBV combination represents a promising treatment in this setting.

The SOLAR-2 study (Table 2) was another open-label Phase II, multicenter, randomized trial that evaluated the efficacy and safety of a fixed-dose combination of LDV/SOF for the treatment of chronic HCV-1 or -4 infection in patients with decompensated cirrhosis or posttransplant recurrence. ${ }^{36}$ The study included 328 patients (more than $90 \%$ were Caucasian, mainly male, mean age 60 years). Most had HCV-1 infection (nearly half with $-1 \mathrm{a}$ and $40 \%$ with $-1 \mathrm{~b}$ ) and 
$10 \%$ had $\mathrm{HCV}-4$ infection while $80 \%$ were TE. All patients were randomized to receive daily LDV/SOF plus RBV for 12 or 24 weeks. One group included 168 posttransplant patients with fibrosis stage F0 to F3 or compensated cirrhosis (ChildPugh A) who were treated for 12 weeks $(n=86)$ or 24 weeks $(\mathrm{n}=82)$. A second group included 160 pre- and posttransplant patients with decompensated cirrhosis (Child-Pugh B or C) treated for 12 weeks $(n=78)$ or 24 weeks $(n=82)$. The SVR12 rates in posttransplant patients without cirrhosis or with compensated cirrhosis were $95 \%$ in the 12 weeks arm and 98\% in the 24 weeks arm. Among pre- and posttransplant patients with decompensated cirrhosis, the SVR12 rates were $85 \%$ in the 12 weeks arm and $88 \%$ in the 24 weeks arm. Among the subgroup of HCV-4-infected patients, SVR12 rates in the group without cirrhosis or with compensated cirrhosis were $10 / 11(91 \%)$ for the 12 weeks arm and $7 / 7$ (100\%) for the 24 weeks of therapy arm. Among patients with more severe liver disease, only 4/7 (57\%) were cured in 12 weeks compared with $6 / 7$ (86\%) in the 24 weeks arm. SVR12 was associated with an improvement of liver function leading to reversion of Child-Pugh classification: from B to A in 35\%, and from $\mathrm{C}$ to $\mathrm{B}$ in $48 \%$ and $\mathrm{A}$ in $5 \%$. The study included patients with impaired liver function and that could explain why serious adverse events were reported in $15 \%$ of ChildPugh A and in 28\% of Child-Pugh B/C patients. LDV/SOF was well-tolerated and treatment discontinuation was very uncommon. There were ten cases of death (three in the posttransplant and seven in the pre/posttransplant groups), but none of the deaths were treatment-related.

In conclusion, a fixed-dose combination of $\mathrm{LDV} / \mathrm{SOF}$ plus RBV provides high SVR12 rates among patients with decompensated cirrhosis or posttransplantation. Patients in SOLAR-1 and -2 achieved comparably high efficacy with the LDV/SOF combination. ${ }^{34,36}$ These patients were not formerly eligible for IFN-containing regimens and the combination treatment reduced the likelihood of recidivism of $\mathrm{HCV}$ infection after organ transplantation.

\section{LDV/SOF in chronic HCV-HIV coinfected patients}

Two studies (one Phase II, the ERADICATE study, and one Phase III, the ION-4 study) (Table 3 ) have been conducted to evaluate the efficacy and safety of the treatment of $\mathrm{CHC}$ in HIV-HCV coinfected patients with LDV/SOF. ${ }^{19,20}$

The ERADICATE study evaluated the rates of SVR following a 12-week treatment regimen of a fixed-dose combination of LDV/SOF in patients coinfected with HCV-1 and included 50 participants, of whom $\sim 80 \%$ had harder-to-treat
Table 3 LDF/SOF in HCV-HIV coinfection: SVRI 2 by subgroup and baseline characteristics

\begin{tabular}{|c|c|c|}
\hline Trial & $\begin{array}{l}\text { Subgroup and baseline } \\
\text { characteristics }(n)\end{array}$ & SVR I 2 rate (\%) \\
\hline \multicolumn{3}{|c|}{ NIAID ERADICATE $^{19}$} \\
\hline & ARV & \\
\hline & Untreated $(n=13)$ & $13(100)$ \\
\hline & Treated $(n=37)$ & $36(97)$ \\
\hline & Overall $(n=50)$ & $49(98)$ \\
\hline \multicolumn{3}{|c|}{$10 N-4^{20}$} \\
\hline & Treatment & \\
\hline & Naïve $(n=150)$ & $142(95)$ \\
\hline & Experienced $(n=\mid 85)$ & $179(97)$ \\
\hline & Cirrhosis & \\
\hline & No $(n=268)$ & $258(96)$ \\
\hline & Yes $(n=67)$ & $63(94)$ \\
\hline & Ethnicity & \\
\hline & Non-Blacks $(n=2 \mid 7)$ & $215(99)$ \\
\hline & Blacks ( $n=|| 5)$ & $103(90)$ \\
\hline & BL CD4 count (cells $/ \mu \mathrm{L})$ & \\
\hline & $<350(\mathrm{n}=37)$ & $35(95)$ \\
\hline & $\geq 350(n=298)$ & $286(96)$ \\
\hline & $A R V$ regimen & \\
\hline & $\mathrm{EFV}+\mathrm{FTC}+\mathrm{TDF}(\mathrm{n}=160)$ & $161(94)$ \\
\hline & $\mathrm{RPV}+\mathrm{FTC}+\operatorname{TDF}(\mathrm{n}=29)$ & $28(97)$ \\
\hline & $\mathrm{RAL}+\mathrm{FTC}+\operatorname{TDF}(\mathrm{n}=146)$ & $|4|$ (97) \\
\hline & Overall $(n=335)$ & $321(96)$ \\
\hline
\end{tabular}

Abbreviations: ARV, antiretroviral; BL, baseline; $E F V$, efavirenz; FTC, emtricitabine; HCV, hepatitis C virus; LDV/SOF, ledipasvir and sofosbuvir; RAL, raltegravir; RPV, rilpirivine; SVRI2, sustained virologic response 12 weeks off therapy; TDF, tenofovir disoproxil furamate.

subtype 1a. ${ }^{19}$ All participants were TN. Most of the patients were men, $>80 \%$ were African-American, and the median age was 58 years. Most had high HCV viral load. About a quarter had advanced liver fibrosis (stage F3), and patients with cirrhosis (stage F4) were excluded.

Patients had well-preserved immune function without signs of AIDS. Thirteen patients were not yet taking antiretroviral therapy (ART) and had both a stable CD4 T-cell count and HIV RNA $<500$ copies/mL or a CD4 cell count $>500$ cells $/ \mathrm{mm}^{3}$ (median 687 cells $/ \mathrm{mm}^{3}$ ). The other 37 were receiving ART with undetectable HIV RNA and a median CD4 count of 576 cells $/ \mathrm{mm}^{3}$. ART consisted of tenofovir/emtricitabine, mostly with efavirenz, raltegravir, or rilpivirine as the third antiretroviral (ARV).

The overall SVR 12 rate was $98 \%$ with $100 \%$ in the ARTuntreated patients, and $97 \%$ in the ART-treated patients. ${ }^{19}$ The single participant with protocol-defined virological failure was a 63-year-old African-American woman with HCV-1b, mild fibrosis, and a CD4 count of 395 cells $/ \mathrm{mm}^{3}$; she had undetectable HCV viral load at the end of 12 weeks of treatment but relapsed 2 weeks later. Deep sequencing 
was conducted and the only mutation detected was a $\mathrm{Y} 93 \mathrm{H}$ mutation in the NS5A region.

LDV/SOF was safe and well-tolerated. The most common adverse events included fatigue, pain, diarrhea, constipation, and headache.

LDV/SOF treatment had no adverse impact on HIV disease: no clinically significant changes in HIV-1 RNA were observed (one ART-treated patient had transient virologic breakthrough associated with nonadherence) and no changes in $\mathrm{CD} 4^{+}$cell count or $\mathrm{CD} 4^{+}$cell percentage were seen during treatment.

The ION-4 Phase III trial evaluated the efficacy and safety of LDV/SOF combination treatment in HCV-HIV coinfected patients receiving ARV therapy. ${ }^{20} \mathrm{HCV}$ TN and TE HIV coinfected patients on stable, approved ARV regimens were enrolled and patients received LDV/SOF once daily for 12 weeks. Patients with compensated cirrhosis were eligible. Permitted concomitant ARV therapy included tenofovir disoproxil furamate (TDF) and emtricitabine with raltegravir (44\%), efavirenz (48\%), or rilpivirine (9\%). The primary efficacy endpoint was SVR12.

In all, 335 patients with genotype $1 \mathrm{a}(75 \%)$, genotype $1 \mathrm{~b}$ (23\%), and genotype 4 (2\%) were enrolled; $82 \%$ were male, and $61 \%$ were White with a mean age of 52 years (range 26-72). The mean baseline HCV RNA was $6.7 \log _{10} \mathrm{IU} / \mathrm{mL}$ (range 4.1-7.8) with a median baseline CD4 count of 628 cells $/ \mathrm{mm}^{3}$. Twenty percent of patients had cirrhosis, $24 \%$ were $I L 28 B$ CC genotype, and 55\% had not responded to prior $\mathrm{HCV}$ treatment. It is interesting to note that $38 \%$ of patients were of Black ethnicity.

The SVR12 rate was $96 \%$ of patients. Two patients had on-treatment virologic failure likely due to noncompliance, ten patients had virologic relapse after discontinuing treatment, and one patient died due to intravenous drug use-related endocarditis/ sepsis. The SVR12 rate was similar in patients with (94\%) and without (96\%) cirrhosis and in TN (94\%) and TE (97\%) patients. ${ }^{20}$ In multivariate analysis, only Black ethnicity was associated with a lower rate of SVR. No specific explanations were available to explain this difference. No differences in pharmacokinetics were observed between patients of Black and non-Black ethnicity. No patient had confirmed HIV virologic rebound (HIV-1 RNA $\geq 400$ copies $/ \mathrm{mL}$ ). Adverse events occurred in $\geq 10 \%$ of patients and included headache (25\%), fatigue $(21 \%)$, and diarrhea $(11 \%)$. No patients discontinued study drug due to an adverse event. No significant laboratory abnormalities were observed..$^{20}$

The IFN-free, RBV-free, single-tablet regimen of LDV/ SOF administered once daily for 12 weeks is, therefore, highly effective and well-tolerated in TN and TE, HCV-1 or -4-infected patients with HIV-1 coinfection, including those with cirrhosis (ION-4 study) and SVR rates are now consistently the same in coinfected and monoinfected patients. The primary consideration when selecting a treatment is not efficacy but potential drug-drug interactions with concomitant medications that could reduce the efficacy or increase the potential toxicity of either the HCV or ARV therapy. The data on drug-drug interactions with currently available medications are still evolving.

At the Conference of Retroviruses and Opportunistic Infections meeting in 2015, German et $\mathrm{al}^{25}$ reported findings from a Phase I study that evaluated interactions between LDV/SOF and ARV regimens containing ritonavir-boosted atazanavir (Reyataz) or darunavir (Prezista) plus tenofovir/ emtricitabine (Truvada) in healthy HIV-negative volunteers.

Drug-drug interaction studies were performed with commonly used ARV regimens. Regimens containing efavirenz (Sustiva), raltegravir (Isentress), or rilpivirine (Edurant) plus Truvada were found to be safe when combined with LDV/SOF, which explains why coinfected participants were limited to these ARV regimens in LDV/SOF trials, such as ION-4 and ERADICATE. ${ }^{19,20,25}$

There is a known drug-drug interaction between LDV and tenofovir, and this drug-drug interaction is compounded when LDV/SOF is administered with the tenofovir prodrug, TDF, plus boosted protease inhibitors, in part because boosted protease inhibitors raise tenofovir levels in patients who are receiving TDF. Data presented by German et $\mathrm{a}^{25}$ indicate that LDV/SOF increases atazanavir, ritonavir, and tenofovir exposure. In turn, atazanavir/ritonavir plus TDF/emtricitabine increases LDV levels. In addition, coadministration of darunavir/ritonavir and TDF/emtricitabine results in modest decreases in SOF levels that are likely not clinically significant.

Concerning hepatitis $\mathrm{C}$ treatment in HIV-HCV coinfected people, current treatment guidelines (EASL and American Association for the Study of Liver Diseases/ Infectious Diseases Society of America) indicate that HIVpositive patients can be treated with the same recommended regimens as HCV monoinfected patients, taking into account potential interactions with ARV therapy. ${ }^{37,38}$

\section{Impact of negative predictors of SVR derived from IFN-based therapy}

From treatment of HCV infection with IFN-based therapy, we have learned that predictors of lower SVR included cirrhosis, IL28B genotype non-CC, Black ethnicity, age, high HCV viral load, and HIV infection. ${ }^{39-41}$ Moreover, IFN-based 
Table 4 SVR rate according to the presence of cirrhosis

\begin{tabular}{|c|c|c|c|c|}
\hline \multirow[t]{3}{*}{ Trial } & \multicolumn{4}{|c|}{ SVR rate by treatment type and treatment duration } \\
\hline & LDV/SOF & LDV/SOF + RBV & LDV/SOF & LDV/SOF + RBV \\
\hline & \multicolumn{2}{|l|}{12 weeks } & \multicolumn{2}{|l|}{24 weeks } \\
\hline \multicolumn{5}{|l|}{ ION-I9 (treatment-naïve) } \\
\hline Noncirrhosis & $179 / 179(100.0)$ & I78/I78 (100.0) & $181 / / 82(99.5)$ & I79/I79 (100.0) \\
\hline Cirrhosis & $32 / 33(97.0)$ & $33 / 33(100.0)$ & $31 / 32(96.9)$ & $36 / 36(100.0)$ \\
\hline Overall & $211 / 213(99.5)$ & $211 / 211(100.0)$ & $212 / 214(99.0)$ & $215 / 215(100.0)$ \\
\hline \multicolumn{5}{|l|}{ ION-2 ${ }^{16}$ (treatment-experienced) } \\
\hline Noncirrhosis & $83 / 87(95.4)$ & $89 / 89(100.0)$ & $86 / 87(100.0)$ & $88 / 89(98.9)$ \\
\hline Cirrhosis & 19/22 (86.4) & $18 / 22(81.8)$ & $22 / 22(100.0)$ & $22 / 22(100.0)$ \\
\hline Overall & $102 / 109(93.6)$ & |07/III (96.4) & $108 / 109(100.0)$ & II0/III (99.I) \\
\hline $\begin{array}{l}\text { Post hoc analysis* of cirrhotic pts } \\
\text { in Phase II and III trials }{ }^{42}\end{array}$ & 109/II8 (92) & 196/204 (96) & 130/I33 (98) & $58 / 58(100)$ \\
\hline
\end{tabular}

Note: *The overall SVRI 2 rate in compensated cirrhotic patients was $493 / 513$ (96\%) with $95 \%$ confidence interval of $94 \%-98 \%$.

Abbreviations: LDV/SOF, ledipasvir and sofosbuvir; pts, patients; RBV, ribavirin; SVRI2, sustained virologic response 12 weeks off therapy.

regimens are often contraindicated in patients with decompensated cirrhosis. From Phase III trials with LDV/SOF (ION studies and Japanese study), the SVR12 was much higher among the subpopulations of HCV-1-infected patients with these negative predictors than expected..$^{9,16,17,27}$ For example, in patients with compensated cirrhosis (Table 4), SVR12 was globally $>95 \%$ in post hoc analysis of Phase II and Phase III trials; ${ }^{42}$ in patients of Black origin (Table 5), SVR12 rates were similar to those in patients of non-Black origin from the ION studies or between Caucasians and Japanese patients; ${ }^{43}$ patients with the non-CC IL28B genotype had comparable SVR12 rates to those with the CC genotype (ION trials); ; 16,17 there was no impact of viral load on SVR12 rates (ION trials), although ION-3 showed that treatment duration could be shortened for patients with HCV viral load $<6$ million $\mathrm{IU} / \mathrm{mL} ;{ }^{17,28}$ patients coinfected with HIV treated with LDV/ SOF had similar SVR12 rates to those who were HCV-1 monoinfected (ERADICATE and ION-4 studies). ${ }^{19,20}$ The ION-4 study indicates that ethnicity may play a role in the SVR rate among HIV-HCV-1 coinfected patients. ${ }^{20}$ These preliminary results need to be evaluated in other studies among HIV-HCV-1 or -4 coinfected patients.

Table 5 Overall SVRI2 rate in Black vs non-Black patients from ION trials

\begin{tabular}{ll}
\hline Ethnicity & SVR rate (\%) \\
\hline Overall $(\mathrm{n}=\mathrm{I}, 95 \mathrm{I})$ & $\mathrm{I}, 887 / \mathrm{I}, 95 \mathrm{I}(97)$ \\
Black $(\mathrm{n}=308)$ & $294 / 308(95)$ \\
Non-Black $(\mathrm{n}=\mathrm{I}, 64 \mathrm{I})$ & $\mathrm{I}, 59 \mathrm{I} / \mathrm{I}, 640 *(97)$ \\
\hline Notes: *Excluding one patient with HCV-4 infection. Reproduced from Wilder JM, \\
Jeffers LJ, Ravendhran N, et al. Safety and efficacy of ledipasvir-sofosbuvir in black patients \\
with hepatitis C virus infection: A retrospective analysis of phase 3 data. Hepatology. \\
Copyright $@ 20$ I5 Wilder et al. HEPATOLOGY published by Wiley Periodicals, Inc., \\
on behalf of the American Association for the Study of Liver Diseases. ${ }^{43}$ \\
Abbreviation: SVRI2, sustained virologic response I2 weeks off therapy.
\end{tabular}

\section{LDV/SOF tolerance and adverse events}

Across the ION $-1,-2$ and -3 studies, treatment with oral LDV/SOF was well-tolerated..$^{9,16,17,44}$ Fewer adverse events were observed in groups who received LDV/SOF alone compared with their counterparts who received RBV. In the RBV-free groups, the most common adverse events were headache and fatigue. In the RBV-regimen groups, the most common adverse events were fatigue, headache, nausea, insomnia, and anemia. Treatment discontinuation due to treatment adverse events was very uncommon in the ION $(-1,-2,-3)$ trials $(13 / 1,518$ [0.8\%]) among HCV-1 monoinfected patients and in the ERADICATE and ION-4 studies among HIV-HCV coinfected patients. ${ }^{9}, 16-20$ Cirrhotic patients (ION-1, -2, and Japanese studies) as well as HIV-coinfected patients (ERADICATE and ION-4 studies) generally tolerated LDV/SOF. ${ }^{9,16-20}$ Serious treatment-related adverse events were identified in SOLAR-1: 9/223 (4.0\%) of posttransplanted patients had a $2 \%$ incidence of treatment-emergent death and 30/108 (27.7\%) of decompensated cirrhotic patients had a $5.5 \%$ incidence of treatment-emergent death. ${ }^{30}$ One death was observed in a cirrhotic patient in the Japanese study. ${ }^{18}$ No direct relationship between these deaths and the treatment was identified.

\section{LDV/SOF combination and perspectives for the treatment of HCV infection}

The LDV/SOF combination represents a new avenue for the treatment of $\mathrm{HCV}$ infection. LDV/SOF can be positioned among the leading drugs for the treatment of $\mathrm{HCV}-1$ infection for several reasons. The drug is generally well-tolerated in 
comparison with the previous standard of care. The LDV/ SOF fixed-dose combination is a single tablet taken orally once a day. Compared to the previous standard of care requiring treatment for 48 weeks, duration of chronic HCV-1 infection treatment with LDV/SOF is just 12 or 24 weeks, as recommended by EASL, American Association for the Study of Liver Diseases, and Infectious Diseases Society of America. ${ }^{37,38}$ Among TN patients with HCV RNA $<6$ million $\mathrm{IU} / \mathrm{mL}$ without cirrhosis, treatment duration can even be reduced to 8 weeks. ${ }^{15,27,45}$ The association of these factors (good tolerance, single oral once daily dose plus shorter course) will likely increase treatment adherence. This factor may explain, in part, why the LDV/SOF combination is associated with a higher cure rate for chronic HCV-1 infection. In addition, there is no need for coadministration of RBV unlike other DAA agents, such as Viekira Pak. ${ }^{13,45}$ Interestingly, traditional predictive factors of poor response to IFN-based therapy, including HIV infection, had no impact on the SVR12 rate in studies using the LDV/SOF combination. ${ }^{9}, 14,16,17,20,27$

Regarding organ dysfunction, the LDV/SOF combination can be prescribed to patients irrespective of the degree of liver function impairment (from Child-Pugh A to Child-Pugh C) and in postliver transplant, whereas IFN-based therapy is contraindicated in HCV-infected patients with cirrhosis ChildPugh B or $\mathrm{C}$ and in posttransplant liver decompensation. Younossi et al showed that the LDV/SOF combination had an excellent impact on patient-reported outcomes among cirrhotic patients with $\mathrm{CHC}$ who were successfully treated. ${ }^{46,47}$ For renal dysfunction, only severe renal insufficiency is a contraindication to LDV/SOF therapy.

Compared to the first generation of HCV protease inhibitors, the LDV/SOF combination has few drug interactions; this advantage leads to a wide range of prescription possibilities among HCV-1 or -4-infected patients with comorbidities. However, coadministration with amiodarone, carbamazepin, phenytoin, rifampicin, rosuvastin, St John's wort, tipranavir, and simeprevir should be avoided, and coadministration with digoxin, dabigatran etexilate, and TDF requires a close monitoring. ${ }^{11,24,25}$

There are some limitations to the wider use of LDV/SOF for HCV treatment. First, its efficacy against HCV-3 was low compared with its efficacy against HCV-1. For HCV-2, efficacy of LDV/SOF is expected to be lower based on the $\mathrm{EC}_{50}$, but large studies supporting this hypothesis are lacking. Second, the drug cannot be prescribed in HCV-1 or -4-infected patients with end-stage kidney disease or those who are being treated with amiodarone for cardiac arrhythmia (a very common disease). Third, although relapse is very uncommon, its occurrence seems to be related, in part, to treatment duration, with 24 weeks being notably better than 12 weeks among TE patients and/or those with severe hepatic impairment. Although, noncompliance was the most common reason for LDV/SOF treatment failure. ${ }^{15}$ Finally, the current cost of LDV/SOF is a major issue. This is difficult to reconcile ethically as the high cost may prevent or limit prescription of this effective drug even in high income countries. Moreover, the drug cannot be prescribed in many regions with the greatest prevalence of $\mathrm{HCV}$ infection because of lower incomes in these areas.

LDV/SOF has been approved by EASL for the treatment of HCV infections involving genotypes 4, 5, and 6. This agreement is probably preemptive and was based on the knowledge of antiviral activity of LDV and SOF across HCV genotypes and based on early results from small studies rather than randomized trials. ${ }^{27,31}$ This combination has been considered as optional for the treatment of HCV-4 or -6 (except -6e) by American Association for the Study of Liver Diseases.

There are some issues requiring further evaluation:

- Addition of a third anti-HCV agent could potentially further reduce the treatment duration and/or avoid the very few relapses that have been observed. ${ }^{10}$

- The impact of HCV NS5A-induced resistance by the LDV/SOF combination either on other HCV NS5Ainhibiting drugs or on the next generation of drugs targeting other HCV protein is unknown.

- The efficacy of the LDV/SOF combination on extrahepatic manifestations of $\mathrm{HCV}$ infection, acute $\mathrm{HCV}-1$ or -4 infection, chronic HCV infection with mixed genotypes, and HIV-HCV-1 or -4 coinfected patients with CD4 count $<500$ cells $/ \mathrm{mm}^{3}$ and HIV RNA $>500$ copies $/ \mathrm{mL}$ is currently unknown. Another question is the eventual impact of ethnicity in SVR12 among HIV-HCV-1 coinfected patients.

- Real-life performance of the LDV/SOF combination outside of clinical trials is unknown and this may need to be evaluated. Indeed, as the use of the LDV/SOF combination grows, new issues will likely be related to treatment adherence and drug-drug interactions due to polypharmacy for the treatment of comorbidities. ${ }^{15}$

In conclusion, a single tablet fixed-dose LDV/SOF combination once daily is highly effective, easy to administer, and represents a major step forward in the fight against $\mathrm{HCV}$ infection. Additional trials using the LDV/SOF combination in various other selected patient populations are required. 


\section{Acknowledgment}

We are grateful to Sandy Field for editing assistance.

\section{Disclosure}

The authors report no conflicts of interest in this work.

\section{References}

1. Seeff LB. Natural history of chronic hepatitis C. Hepatology. 2002; 36(suppl 1):S35-S46.

2. Brown RS Jr. Universal hepatitis C eradication prior to liver transplantation: we can do it, but should we? Am J Transplant. 2015;15(7): 1741-1742.

3. Ghany MG, Strader DB, Thomas DL, Seeff LB; American Association for the Study of Liver Diseases. Diagnosis, management, and treatment of hepatitis C: an update. Hepatology. 2009;49(4):1335-1374.

4. Jayasekera CR, Barry M, Roberts LR, Nguyen MH. Treating hepatitis C in lower-income countries. N Engl J Med. 2014;370(20):1869-1871.

5. Gutierrez JA, Lawitz EJ, Poordad F. Interferon-free, direct-acting antiviral therapy for chronic hepatitis C. J Viral Hepat. Epub 2015 Jun 17.

6. Jazwinski AB, Muir AJ. Direct-acting antiviral medications for chronic hepatitis C virus infection. Gastroenterol Hepatol (N Y). 2011; 7(3):154-162.

7. Cooke GS, Hill AM. Diagnostics for resource-limited settings in the era of interferon-free HCV therapy. J Viral Hepat. 2015;22(5):459-460.

8. Lawitz E, Sulkowski MS, Ghalib R, et al. Simeprevir plus sofosbuvir, with or without ribavirin, to treat chronic infection with hepatitis $\mathrm{C}$ virus genotype 1 in non-responders to pegylated interferon and ribavirin and treatment-naive patients: the COSMOS randomised study. Lancet. 2014;384(9956):1756-1765

9. Afdhal N, Zeuzem S, Kwo P, et al. Ledipasvir and sofosbuvir for untreated HCV genotype 1 infection. N Engl J Med. 2014;370(20): 1889-1898.

10. Gentile I, Buonomo AR, Zappulo E, Coppola N, Borgia G. GS-9669: a novel non-nucleoside inhibitor of viral polymerase for the treatment of hepatitis C virus infection. Expert Rev Anti Infect Ther. 2014;12(10) 1179-1186.

11. Harvoni (ledipasvir and sofosbuvir) tablets for oral use [US package insert]. Foster City, CA: Gilead Sciences Inc; 2015.

12. Harvoni (ledipasvir/sofosbuvir) film-coated tablets [EU summary of product characteristics]. Foster City, CA: Gilead Sciences Inc; 2014.

13. Gritsenko D, Hughes G. Ledipasvir/sofosbuvir (Harvoni): improving options for hepatitis C virus infection. P T. 2015;40(4):256-276.

14. Keating GM. Ledipasvir/sofosbuvir: a review of its use in chronic hepatitis C. Drugs. 2015;75(6):675-685.

15. Fazel Y, Lam B, Golabi P, Younossi Z. Safety analysis of sofosbuvir and ledipasvir for treating hepatitis C. Expert Opin Drug Saf. 2015;14(8): $1317-1326$.

16. Afdhal N, Reddy KR, Nelson DR, et al. Ledipasvir and sofosbuvir for previously treated HCV genotype 1 infection. $N$ Engl J Med. 2014;370(16): 1483-1493.

17. Kowdley KV, Gordon SC, Reddy KR, et al. Ledipasvir and sofosbuvir for 8 or 12 weeks for chronic HCV without cirrhosis. N Engl J Med. 2014; 370(20):1879-1888.

18. Mizokami M, Yokosuka O, Takehara T, et al. Ledipasvir and sofosbuvir fixed-dose combination with and without ribavirin for 12 weeks in treatment-naive and previously treated Japanese patients with genotype 1 hepatitis C: an open-label, randomised, phase 3 trial. Lancet Infect Dis. 2015;15(6):645-653.

19. Osinusi A, Townsend K, Kohli A, et al. Virologic response following combined ledipasvir and sofosbuvir administration in patients with HCV genotype1 and HIV co-infection. JAMA. 2015;313(12):1232-1239.

20. Naggie S, Cooper C, Saag M, et al. Ledipasvir and sofosbuvir for HCV in patients coinfected with HIV-1. N Engl J Med. 2015;373(8):705-713.
21. Mogalian E, Mathias A, Yang JC, et al. The pharmacokinetics of ledipasvir, an HCV specific NS5A inhibitor in HCV-uninfected subjects with severe renal impairment [Abstract 1952]. Hepatology. 2014;60 (4 Suppl):1145A-1146A.

22. German P, Mathias A, Yang JC, et al. The pharmacokinetics of ledipasvir, an HCV specific NS5A inhibitor in HCV-uninfected subjects with moderate and severe hepatic impairment [Abstract 467]. Hepatology. 2013;58(4 Suppl):432A.

23. Kirby B, German P, Sheen G, et al. No differences in the pharmacokinetics of sofosbuvir (GS-7977) and sofosbuvir/ledipasvir (GS-7977/ GS-5885) fixed dose combination between Japanese and Caucasian [Abstract 954]. Hepatol Int. 2013;Suppl 1:S375-S376.

24. German P, Garrison K, Pang PS, et al. Drug-drug interactions profile of the fixed-dose combination tablet ledipasvir/Sofosbuvir [Abstract 1976]. Hepatology. 2014;60(4 Suppl):1162A.

25. German P, Garrison K, Pang PS, et al. Drug-drug interactions between anti-HCV regimen ledipasvir/sofosbuvir and antiretrovirals [Abstract 82]. In: 2015 Conference on Retroviruses and Opportunistic Infections; 23-26 February, 2015; Seattle.

26. Mathias A, Comprost M, Clemons D, Denning J, Symonds WT. No clinically significant pharmacokinetic drug-drug interactions between sofosbuvir (GS-7977) and immunosuppressants, cyclosporine A or tacrolimus in healthy volunteers [Abstract 1869]. Hepatology. 2012;56 (4 Suppl):1063A-1064A.

27. Jacobson IM, Kwo PY, Kowdley KV, et al. Virologic response rates to all oral fixed-dose combination ledipasvir/sofosbuvir regimens are similar in patients with and without traditional negative predictive factors in phase 3 clinical trials [Abstract 1945]. Hepatology. 2014;60 (4 Suppl):1141A-1142A.

28. Abergel A, Loustaud-Ratti V, Metivier S, et al. Ledipasvir/sofosbuvir treatment results in high SVR rates in patients with chronic genotype 4 and 5 HCV infection [Abstract 0056]. J Hepatol. 2015;62(Suppl): S219-S220.

29. Gane EJ, Hyland RH, An D, et al. Ledipasvir/sofosbuvir fixed-dose combination is safe and effective in difficult-to-treat populations including GT3 patients, decompensated GT1 patients, and GT1 patients with prior sofosbuvir experience [Abstract 06]. In: 49th Annual Meeting of the European Association for the Study of the Liver; 9-13 April 2014; London.

30. Gane E, Hyland R, An D et al. Efficacy of ledipasvir and sofosbuvir, with or without ribavirin, for 12 weeks in patients with $\mathrm{HCV}$ genotype 3 or 6 infection. Gastroenterology. 2015;149(6):1454-1461.

31. Koli A, Kapoor R, Sims Z, et al. Ledipasvir and sofosbuvir for hepatitis C genotype 4: a proof-of-concept, single-center, open-label phase 2a cohort study. Lancet Infect Dis. 2015;15(9):1049-1054.

32. Smith MA, Mohammad R. Ledipasvir-sofosbuvir for hepatitis C genotype 4 infection. Lancet Infect Dis. 2015;15(9):993-995.

33. Ferenci P. Treatment of hepatitis $\mathrm{C}$ in difficult-to-treat patients. Nat Rev Gastroenterol Hepatol. 2015;12(5):284-292.

34. Charlton M, Everson GT, Flamm SL, et al. Ledipasvir and sofosbuvir plus ribavirin for treatment of $\mathrm{HCV}$ infection in patients with advanced liver disease. Gastroenterology. 2015;149(3):649-659.

35. Kwo PY, Mantry PS, Coakley E, et al. An interferon-free antiviral regimen for HCV after liver transplantation. N Engl J Med. 2014;371(25): 2375-2382.

36. Manns M. Ledipasvir/sofosbuvir with ribavirin is safe and efficacious in decompensated and post liver transplantation patients with $\mathrm{HCV}$ infection: preliminary results of the prospective SOLAR 2 trial [Abstract G01]. In: EASL 2015. 50th Annual Meeting of the European Association for the Study of the Liver, April 22-26, 2015; Vienna, Austria.

37. American Association for the Study of Liver Diseases/Infectious Diseases Society of America. Recommendations for Testing, Managing, and Treating Hepatitis C. Available from: http://www.hcvguidelines. org/full-report/unique-patient-populations-patients-hivhcv-coinfection. Accessed April 6, 2015.

38. EASL Recommendations on Treatment of Hepatitis C 2015. J Hepatol. 2015;63(1):199-236. 
39. Zeuzem S. Heterogeneous virologic response rates to interferon-based therapy in patients with chronic hepatitis $\mathrm{C}$ : who responds less well? Ann Intern Med. 2004;140(5):370-381.

40. Rodriguez-Torres M, Jeffers LJ, Sheikh MY, et al. Peginterferon alpha-2a and ribavirin in Latino and Non-Latino whites with hepatitis C. N Engl J Med. 2009;360(3):257-267.

41. Nkuize M, Mulkay JP, Adler M, et al. Response of Black African patients with hepatitis $\mathrm{C}$ virus genotype 4 to treatment with peg-interferon and ribavirin. Acta Gastroenterol Belg. 2013;76(3):291-299.

42. Reddy KR, Bourlière M, Sulkowski M, et al. Ledipasvir and sofosbuvir in patients with genotype 1 hepatitis $\mathrm{C}$ virus infection and compensated cirrhosis: an integrated safety and efficacy analysis. Hepatology. 2015;62(1):79-86.

43. Wilder JM, Jeffers LJ, Ravendhran N, et al. Safety and efficacy of ledipasvir-sofosbuvir in black patients with hepatitis $\mathrm{C}$ virus infection: A retrospective analysis of phase 3 data. Hepatology. 2016;63(2):437-444.
44. Alqahtani S, Afdhal NH, Zeuzem S, et al. Safety of ledipasvir/sofosbuvir with and without ribavirin for the treatment of patients with chronic HCV genotype 1 infection: an analysis of the phase 3 ION trials. Hepatology. 2015;62(1):25-30.

45. Gentile I, Borgia G. Ledipasvir/sofosbuvir administration achieves very high rate of viral clearance in patients with HCV genotype 1 infection without cirrhosis, regardless of ribavirin co-administration or length of treatment. Evid Based Med. 2014;19(6):223-224.

46. Younossi ZM, Stepanova M, Pol S, Bronowicki JP, Carrieri MP, Bourlière $M$. The impact of ledipasvir/sofosbuvir on patient-reported outcomes in cirrhotic patients with chronic hepatitis C: the SIRIUS study. Liver Int. Epub 2015 Jun 9.

47. Bourlière M, Bronowicki JP, de Ledinghen V, et al. Ledipasvirsofosbuvir with or without ribavirin to treat patients with HCV genotype 1 infection and cirrhosis non-responsive to previous protease-inhibitor therapy: a randomised, double-blind, phase 2 trial (SIRIUS). Lancet Infect Dis. 2015;15(4):397-404.
Therapeutics and Clinical Risk Management

\section{Publish your work in this journal}

Therapeutics and Clinical Risk Management is an international, peerreviewed journal of clinical therapeutics and risk management, focusing on concise rapid reporting of clinical studies in all therapeutic areas outcomes, safety, and programs for the effective, safe, and sustained use of medicines. This journal is indexed on PubMed Central, CAS,

\section{Dovepress}

EMBase, Scopus and the Elsevier Bibliographic databases. The manuscript management system is completely online and includes a very quick and fair peer-review system, which is all easy to use. Visit http://www.dovepress.com/testimonials.php to read real quotes from published authors. 